\title{
Developing the Patient Health Questionnaire-8 for a greater impact on the quality of life of patients with functional dyspepsia compared to Somatic Symptom Scale-8
}

Chaoqun Yuan ${ }^{1 \dagger}$, Guizhen Yong ${ }^{2 \dagger}$, Xi Wang $^{1}$, Ting Xie ${ }^{1}$, Chunyan Wang ${ }^{1}$, Yuan Yuan ${ }^{1}$ and Guobin He ${ }^{1 *}$ (D)

\begin{abstract}
Background: To develop the Patient Health Questionnaire-8 (PHQ-8) as a more reliable approach than the Somatic Symptom Scale-8 (SSS-8), evaluating somatization which might be a critical factor influencing the quality of life (QoL) in patients with functional dyspepsia (FD). Also, the effects of somatization on QoL of FD patients were assessed by these two approaches.

Methods: Herein, 612 FD patients completed a questionnaire involving 25 items. 8/25 items were selected to develop the PHQ-8 by four methods of discrete degree, correlation coefficient, factor analysis, and Cronbach's a coefficient. Reliability and validity of the PHQ-8 and the SSS-8 were compared by principal component and confirmatory factor analyses. The effects of somatization, depression, and anxiety on the Nepean Dyspepsia Index (NDI) for QoL were explored by Pearson's correlation coefficient and linear regression analysis.

Results: The Cronbach's a coefficient for the PHQ-8 and the SSS-8 was 0.601 and 0.553 , respectively, and the cumulative contribution rate of three extracted factors for the developed PHQ-8 and SSS-8 was $55.103 \%$ and $51.666 \%$, respectively. Somatization evaluated by the PHQ-8 $(r=0.309, P<0.001)$ and the SSS-8 $(r=0.281, P<0.001)$ was found to be correlated to NDI. The model used for the PHQ-8 showed that the values of goodness-of-fit index (GFI) and adjusted GFI (AGFI) were 0.984 and 0.967 , respectively, which indicated that the model fitted well. Linear regression analysis unveiled that somatization $(\beta=0.270, P<0.001)$, anxiety $(\beta=0.163, P<0.001)$, and depression $(\beta=0.136$, $P=0.003$ ) assessed by the PHQ-8 were correlated to NDI. In addition, somatization ( $\beta=0.250, P<0.001)$, anxiety ( $\beta=0.156, P<0.001)$, and depression $(\beta=0.155, P=0.001)$ evaluated by the SSS- 8 were correlated to NDI.
\end{abstract}

Conclusions: PHQ-8 showed a superior reliability and validity, and somatization assessed by the developed PHQ-8 showed a greater influence on the QoL of FD patients as compared to the SSS-8. Our findings suggested that the

\footnotetext{
*Correspondence: heguob@163.com

${ }^{\dagger}$ Chaoqun Yuan and Guizhen Yong contributed equally to this work

1 Department of Gastroenterology, Affiliated Hospital of North Sichuan

Medical College, No. 67 Wenhua Road, Shunqing, Nanchong 637000,

Sichuan, China

Full list of author information is available at the end of the article
}

(C) The Author(s) 2020. Open Access This article is licensed under a Creative Commons Attribution 4.0 International License, which permits use, sharing, adaptation, distribution and reproduction in any medium or format, as long as you give appropriate credit to the original author(s) and the source, provide a link to the Creative Commons licence, and indicate if changes were made. The images or other third party material in this article are included in the article's Creative Commons licence, unless indicated otherwise in a credit line to the material. If material is not included in the article's Creative Commons licence and your intended use is not permitted by statutory regulation or exceeds the permitted use, you will need to obtain permission directly from the copyright holder. To view a copy of this licence, visit http://creativecommons.org/licenses/by/4.0/. The Creative Commons Public Domain Dedication waiver (http://creativeco mmons.org/publicdomain/zero/1.0/) applies to the data made available in this article, unless otherwise stated in a credit line to the data. 
developed PHQ-8 may show improvement in a reliable assessment of the effects of somatization on FD patients in lieu of the SSS-8.

Keywords: Patient health questionnaire-8, Functional dyspepsia, Somatization, Somatic Symptom Scale-8, Reliability, Validity, Quality of life

\section{Background}

Pathogenesis and subtypes of functional dyspepsia

Functional dyspepsia (FD) is characterized by bothersome epigastric pain or burning, postprandial fullness, or early satiation without evidence of structural disease. According to the statistics, the prevalence of FD is as high as $20-30 \%[1,2]$. The underlying pathogenesis includes diverse mechanisms, such as infectious causes represented by Helicobacter pylori (H. pylori) [1-6], diet factor $[1,7,8]$, gastric acid $[1,9,10]$, delayed gastric emptying, impaired proximal gastric accommodation [1, 11-13], visceral hypersensitivity [1, 14-16], duodenal inflammation $[1,17,18]$, genetic factors $[19,20]$, and psychosocial factors (such as anxiety, depression, and stress) [19-25]. Moreover, these factors may interact with each other under the participation of brain-gut axis $[1,3,15]$. Thus, FD is a disorder of gut-brain interaction and classified into three subtypes based on Rome IV criteria: (1) epigastric pain syndrome (EPS): upper abdominal pain and/or burning discomfort of upper abdomen; (2) postprandial distress syndrome (PDS): postprandial fullness and early satiety; (3) the overlapped group of EPS and PDS [2].

\section{FD patients with common somatization symptoms}

In addition, FD patients often have dizziness, back pain, sleep disorders, fatigue [26], and other symptoms of digestive system that cannot be explained by biochemical and structural abnormalities. Clinically, these symptoms are known as somatization symptoms [27, 28]. Somatization is defined as a chronic mental disorder characterized by the presence of one or more frequently changing somatic symptoms, involving multiple systems and organs of the body [29]. Those symptoms often induce patients' incorrect understanding or excessive attention, imposing a huge economic burden on the society [30]. Somatization can coexist with other medical disorders, such as anxiety and depression, rendering them complex and changeable [31]. Additionally, it affects the severity of dyspepsia and the quality of life (QoL) of FD patients [32, 33]. Somatization plays a more significant role in dyspepsia symptom severity (DSS) as compared to that in gastric sensitivity, anxiety, and depression in FD patients [21]. It is an independent risk factor for impaired QoL of FD patients, and a 5-year follow-up study demonstrated that proximal gastric accommodation, gastric emptying, and $H$. pylori infection were not found as risk factors [33]. Therefore, assessment of somatization is highly essential for studying FD patients.

\section{Limitations of questionnaires for somatization}

The symptoms of somatization disorder were widely assessed by the Patient Health Questionnaire-15 (PHQ15) developed by Kroenke et al. [34]. However, PHQ-15 includes a number of items overlapped with gastrointestinal symptoms in FD patients. Therefore, symptoms of somatization disorder have been investigated by the Patient Health Questionnaire-12 (PHQ-12) with the removal of three gastrointestinal symptoms, while both PHQ-12 and PHQ-15 had dysmenorrhea or other items related to menstrual discomfort, making the gender score different [34-36].

On the other hand, symptoms of somatization disorder were evaluated by the Somatic Symptom Scale-8 (SSS-8) with satisfactory reliability and validity developed in the field of the Diagnostic and Statistical Manual of Mental Disorders (Fifth Edition) (DSM-5) [37-39]. Therefore, it was previously recommended to be a replacement for the PHQ-15 [40]. However, the SSS-8 is not a FD-specific scale for evaluating symptoms of somatization disorder, in which the relevant items may overlap with gastrointestinal symptoms. Subsequently, we developed the Patient Health Questionnaire-8 (PHQ-8) for assessing FD patients with symptoms of somatization disorder by adapting SSS-8, and confirmed its reliability and validity. The PHQ-8 had a greater influence on the QoL of FD patients than the SSS-8.

\section{Patients and methods}

\section{Patients}

From June 2017 to June 2018, patients who were suspected of FD were admitted to the Department of Gastroenterology in Affiliated Hospital of North Sichuan Medical College. According to CONSORT guidelines, inclusion criteria were as follows: (1) patients who met Rome IV criteria [1], presented upper abdominal pain, a burning feeling in the upper stomach, postprandial fullness, and early satiety of one or more symptoms, but no evidence of organic diseases related to the above-mentioned symptoms; existence of these symptoms for at least 6 months, as well as satisfying the Rome IV criteria for at least 3 months; (2) patients aged $\geq 18$-years-old; 
(3) patients whose level of education was primary school and above, and had a certain reading comprehension ability. Exclusion criteria were as follows: (1) patients with organic gastrointestinal diseases, including erosive esophagitis and Barrett's esophagus, which was diagnosed by gastroscopy; (2) patients with systemic diseases, metabolic diseases, or malignant tumors diagnosed by B-scan ultrasound, blood routine, and biochemical examinations; (3) patients with irritable bowel syndrome (IBS); (4) patients with gastroesophageal reflux disease with symptoms of acidic taste in the mouth, regurgitation, and heartburn; (5) having a history of abdominal surgery, such as cholecystectomy, intestinal resection, hysterectomy, or appendectomy; (6) patients who had recently ingested anticholinergic drugs, antispasmodic and analgesic drugs, hormones, and nonsteroidal and antiinflammatory drugs; patients whose upper abdominal symptoms disappeared after eradicating $H$. pylori infection; (7) pregnant and lactating women; or (8) patients with psychosis and serious somatic diseases (anxiety and depression). The sample size was 395 patients in a developed IBS-specific somatization scale [27]. A minimum sample size of 300 patients achieved a 95\% confidence interval to equally split chance (50/50), according to the formula, wherein 350 cases were studied [41]. Herein, we used a sample size of more than 400 patients. While developing PHQ-8, FD patients accompanied with anxiety and depression were excluded to avoid the interaction effects of anxiety, depression, and somatization disorders. However, previous studies showed that anxiety, depression, and somatization had mutual effects [21, 33]. Therefore, in the clinical application of the developed PHQ-8, FD patients with anxiety and depression could be included. All the patients signed the written informed consent form.

\section{Pool items for the development of the PHQ-8}

According to SSS-8, PHQ-15 items and the 25 most common clinical symptoms of somatization disorder were involved in the pool items: 1 . back pain; 2. pain in arms, legs, or joints; 3 . dysmenorrhea or menstrual other discomfort; 4. headaches; 5 . chest pain; 6 . shortness of breath; 7. dizziness; 8 . fainting; 9. palpitation; 10 . sexual life pain or other discomfort; 11 . feeling tired or having low energy; 12. insomnia or other sleep problems; 13. numbness/stinging; 14 . fatigue; 15 . throat discomfort (foreign body sensation/dryness/pain); 16. dry mouth; 17. excessive sweat; 18. hand/foot heavy feeling; 19. a burst of cold/fever; 20. memory loss/forgetfulness; 21. urinary frequency; 22. urinary pain/dysuria; 23. blurred vision; 24. neck and shoulder pain; 25. muscle pain. Interestingly, 1-12 items were included in the original PHQ15 [35], in which each item was scored by three-level as 0 ("not bothered at all"), 1 ("slightly bothered"), or 2 ("remarkably bothered"). All FD patients completed a questionnaire involving 25 items by self. The items of the developed PHQ-8 were completed by discrete degree method, correlation coefficient method, factor analysis method, and Cronbach's $\alpha$.

All enrolled patients filled in the PHQ-15, the SSS-8, the Generalized Anxiety Disorder-7 (GAD-7) [42] for anxiety, the Patient Health Questionnaire (PHQ-9) [43] for depression, the DSS [33], and the Nepean Dyspepsia Index-Short Form (NDI) [44] for QoL.

\section{Items were selected by the four methods for the development of the PHQ-8}

Discrete degree method: Since the low degree of dispersion of the selected item revealed poor ability of evaluation, the items with high degree of dispersion were selected, and the degree of dispersion was measured by the standard deviation (SD) of scores of items, in which exclusion of the SD of the items was defined as $<0.5$ [45].

Correlation coefficient method: An item with a correlation coefficient $>0.3$ was selected according to Cohen's criteria [39].

Factor analysis with the principal component method: The common factor was extracted according to the feature root $>1$, and the variance was maximized by orthogonal rotation to select an item with a factor load $>0.4$. The Kaiser-Meyer-Olkin (KMO) and Bartlett's spherical tests were performed. The value of $\mathrm{KMO}<0.5$ is unsuitable for factor analysis, and in Bartlett's spherical test, $P<0.01$ can negate the zero hypothesis that the correlation matrix is a unit matrix, i.e., a significant correlation was established between the variables [46].

Cronbach's $\alpha$ coefficient: The items were screened for internal consistency, and the Cronbach's $\alpha$ coefficient of the initial total scale was calculated. If the Cronbach's $\alpha$ coefficient increased after the deletion of an item, it can be concluded that the existence of an item may reduce the internal consistency [47].

\section{DSS}

The DSS score was calculated as the sum of all eight symptoms: postprandial fullness, early satiation, epigastric pain, burning, bloating, belching, nausea, and vomiting by a 3-point scale (absent, mild, moderate, severe) [33]. The correlation among the DSS, PHQ- 8 , and SSS-8 was evaluated, and the effects of somatization, anxiety, and depression disorders on DSS were analyzed.

\section{Evaluation of psychological disorder}

The PHQ-15 questionnaire was designed for somatization disorder, in which the severity of 15 symptoms was rated for the last 4 weeks by three-level as 0 ("not 
bothered at all"), 1 ("slightly bothered"), or 2 ("remarkably bothered") [34]. The SSS-8, composed of 8 somatic symptoms, was also applied by three-level as 0 ("not bothered at all"), 1 ("slightly bothered"), or 2 ("remarkably bothered") [38]. The Generalized Anxiety Disorder-7 (GAD-7) was utilized to measure the severity of GAD and diagnosis comorbidity in GAD (total score $>10$-point) [42]. The PHQ-9 was employed to identify major depressive disorder (total score $>10$-point), and nine items were rated on a 3-point Likert scale (0-3) [43].

\section{Measurement of disease-specific QoL}

The Short-Form Nepean Dyspepsia Index (SF-NDI) was used to measure FD patients' QoL: inference with work/ study, tension, inference with daily activities, disputation to daily eating/drinking, knowledge toward/control over disease symptoms over the past 2 weeks. Each item was assessed on a 5-point scale from 0 (not at all), 1 (slightly), 2 (moderately), 3 (remarkably) to 4 (extremely) [44]. The correlations among the SF-NDI, the PHQ-8, and the SSS- 8 as well as the effects of somatization, depression, and anxiety disorders on FD patients' QoL were assessed.

\section{Statistical analysis}

SPSS 24.0 software (IBM, Armonk, NY, USA) was utilized to carry out statistical analysis. Quantitative data were expressed as mean $\pm \mathrm{SD}$, t-test was used for making comparison between two groups, and one-way analysis of variance (ANOVA) was used for comparing more than two groups. The qualitative data were presented by rate, and chi-square test was used for making comparison. Moreover, AMOS 22.0 software was applied for confirmatory factor analysis. Pearson's correlation coefficient was used for correlation analysis. Additionally, factors influencing NDI, such as somatization, anxiety, and depression, were analyzed by linear regression analysis; $P<0.05$ was considered statistically significant.

\section{Results}

Demographic characteristics and somatization scores of FD patients

Herein, 612 patients were diagnosed with FD according to Rome IV criteria. $17.3 \%(106 / 612)$ of the patients were identified with anxiety disorders, $13.6 \%(83 / 612)$ had comorbidity of depression, and $7.8 \%$ (48/612) were diagnosed with comorbidity of anxiety and depression. Among $471 \mathrm{FD}$ patients without comorbidity of anxiety and depression, $63.5 \%(299 / 471)$ and 36.5\% (172/471) of the patients were female and male, respectively. The proportion of FD patients' level of education for primary school, middle school, high school, college or above was $22.7 \%$ (107/471), $47.3 \%$ (223/471), $14.4 \%$ (68/471), and $15.5 \%$ (73/471), respectively. The mean age of the patients was $43.4 \pm 10.3$ (range 18-67)-years-old. Moreover, $20.6 \%(97 / 471)$ of the patients were diagnosed with EPS, $31.0 \%(146 / 471)$ had PDS, and 48.4\% (228/471) showed both EPS and PDS. The FD patients' demographic characteristics used for PHQ- 8 and the SSS- 8 are shown in Table 1.

Among the scores evaluated by these two scales, a significant difference was found in gender $(P<0.05)$. However, no significant differences were detected between different ages and levels of education $(P<0.05)$.

\section{Items used for developing the PHQ-8 were selected} by the four methods

Using discrete degree method, chest pain, shortness of breath, fainting, palpitation, sexual life pain or other

Table 1 Demographic characteristics of 471 FD patients versus scores of two somatization scales (mean \pm SD)

\begin{tabular}{|c|c|c|c|c|c|c|}
\hline & PHQ-8 & $t / F$ & $P$ value & SSS-8 & $t / F$ & $P$ value \\
\hline Gender & & 2.484 & 0.013 & & 3.322 & 0.001 \\
\hline Male $(n=172)$ & $4.32 \pm 2.78$ & & & $4.30 \pm 2.29$ & & \\
\hline Female $(n=299)$ & $4.97 \pm 2.65$ & & & $5.04 \pm 2.39$ & & \\
\hline Age (years) & & 1.439 & 0.231 & & 1.987 & 0.115 \\
\hline $18-29(n=45)$ & $3.96 \pm 2.49$ & & & $3.98 \pm 2.03$ & & \\
\hline $30-44(n=201)$ & $4.78 \pm 2.61$ & & & $4.84 \pm 2.33$ & & \\
\hline $45-59(n=211)$ & $4.87 \pm 2.85$ & & & $4.90 \pm 2.48$ & & \\
\hline$\geq 60(n=14)$ & $4.57 \pm 2.62$ & & & $4.50 \pm 2.47$ & & \\
\hline Level of education & & 1.669 & 0.173 & & 2.428 & 0.065 \\
\hline Primary school $(n=107)$ & $5.15 \pm 2.96$ & & & $5.25 \pm 2.76$ & & \\
\hline Junior high school $(n=223)$ & $4.46 \pm 2.71$ & & & $4.54 \pm 2.24$ & & \\
\hline High school $(n=68)$ & $4.87 \pm 2.63$ & & & $4.96 \pm 2.48$ & & \\
\hline College or above $(n=73)$ & $4.82 \pm 2.38$ & & & $4.62 \pm 2.03$ & & \\
\hline
\end{tabular}


discomfort, numbness/tingling, excessive sweat, heavy hands/foot, a bursts of cold/fever, memory loss/forgetfulness, urinary frequency, urinary pain/dysuria, blurred vision, neck and shoulder pain, and muscle pain were removed since the SD of the items was defined as $<0.5$.

Using correlation coefficient method, fainting $(r=0.090)$, dysmenorrhea or menstrual other discomfort $(r=0.243)$, excessive sweat $(r=0.296)$, and urinary pain / dysuria $(r=0.260)$ were removed.

According to factor analysis method, the KMO value for the initial scale was 0.668, and the value of Bartlett's spherical test was 366.894 $(P<0.01)$, which was appropriate for factor analysis, and indicated that each item had a factor loading of $>0.4$ in the dimension except for headaches and chest pain.

Cronbach's $\alpha$ coefficient method showed that the $\alpha$ coefficient increased after excluding fainting and urinary pain/dysuria.

The screened items of the developed PHQ- 8 are presented in Table 2, including back pain, pain in arms, legs, or joints, dizziness, fatigue, dry mouth, feeling tired or having low energy, insomnia or other sleep problems, and throat discomfort.

\section{Reliability analysis of the developed PHQ-8 and the SSS-8 Intrinsic reliability analysis}

Cronbach's $\alpha$ is a measure of the internal consistency or reliability. The test is utilized to examine the consistency and stability of the questionnaires. Hence, the Cronbach's $\alpha$ was applied to ascertain whether the items were reliable in measuring the same dimension. It is speculated that when the Cronbach's $\alpha$ coefficient is $>0.7$, the reliability is satisfactory [48]. The Cronbach $\alpha$ coefficient of the developed PHQ- 8 and the SSS- 8 was 0.601 and 0.553 , respectively. The correlation coefficient between each item and the total score was $0.426-0.652$ and 0.359 0.573 , respectively.

\section{Criterion validity}

The criterion validity unveiled that the correlation coefficient between the total score of the PHQ- 8 and the SSS-8 and the PHQ-15 was $(r=0.739, P<0.001)$ and $(r=0.835$,

Table 2 PHQ-8 developed by screening 25 items using four methods

\begin{tabular}{|c|c|c|c|c|}
\hline Items & Degree of dispersion & $\begin{array}{l}\text { Correlation } \\
\text { coefficient }\end{array}$ & Factor analysis & $\begin{array}{l}\text { Cronbach's a } \\
\text { coefficient }\end{array}$ \\
\hline Back pain & 0.679 & 0.427 & 0.722 & 0.746 \\
\hline Pain in arms, legs, or joints & 0.557 & 0.403 & 0.673 & 0.746 \\
\hline Dysmenorrhea or menstrual other discomfort & $0.464(\times)$ & $0.243(\times)$ & 0.617 & $0.754(\times)$ \\
\hline Headaches & 0.577 & 0.403 & $0.380(\times)$ & 0.746 \\
\hline Chest pain & $0.496(x)$ & 0.395 & $0.362(\times)$ & 0.745 \\
\hline Shortness of breath & $0.497(\times)$ & 0.448 & 0.427 & 0.742 \\
\hline Dizziness & 0.581 & 0.490 & 0.650 & 0.739 \\
\hline Fainting & $0.112(x)$ & $0.090(x)$ & 0.609 & $0.755(\times)$ \\
\hline Palpitation & $0.480(x)$ & 0.386 & 0.514 & 0.746 \\
\hline Sexual pain or other discomfort & $0.294(\times)$ & 0.313 & 0.511 & 0.749 \\
\hline Feeling tired or having low energy & 0.687 & 0.543 & 0.731 & 0.735 \\
\hline Insomnia or trouble sleeping & 0.694 & 0.378 & 0.623 & 0.751 \\
\hline Numbness/tingling & $0.470(x)$ & 0.341 & 0.731 & 0.749 \\
\hline Fatigue & 0.628 & 0.509 & 0.780 & 0.738 \\
\hline Throat discomfort & 0.763 & 0.457 & 0.434 & 0.746 \\
\hline Dry mouth & 0.674 & 0.375 & 0.636 & 0.751 \\
\hline Excessive sweat & $0.466(x)$ & $0.296(\times)$ & 0.757 & 0.751 \\
\hline Hand/foot heavy feeling & $0.364(\times)$ & 0.322 & 0.409 & 0.749 \\
\hline A burst of cold/fever & $0.447(\times)$ & 0.464 & 0.505 & 0.741 \\
\hline Memory loss/forgetfulness & $0.493(\times)$ & 0.415 & 0.724 & 0.744 \\
\hline Urinary frequency & $0.449(x)$ & 0.364 & 0.717 & 0.747 \\
\hline Urinary pain/dysuria & $0.242(x)$ & $0.260(x)$ & 0.740 & 0.751 \\
\hline Blurred vision & $0.419(\times)$ & 0.309 & 0.739 & 0.750 \\
\hline Neck and shoulder pain & $0.487(x)$ & 0.382 & 0.645 & 0.746 \\
\hline Muscle pain & $0.374(x)$ & 0.396 & 0.729 & 0.745 \\
\hline
\end{tabular}

PHQ-8 Patient Health Questionnaire- $8, \times$ indicated that the item was removed 
$P<0.001$ ), respectively. As shown in Table 3 , the developed PHQ-8 outperformed the SSS-8.

\section{Structural validity analysis for the developed PHQ-8 and the SSS-8}

Exploratory factor analysis: The values of developed PHQ-8 were 0.668 and 366.894, $(P<0.01)$ in $\mathrm{KMO}$ and Bartlett's spherical tests, respectively, while those for the SSS-8 were 0.680 and $236.445(P<0.01)$, respectively. The exploratory factor analysis was carried out on the scale, and the principal component method was used to maximize the orthogonal rotation through the covariance matrix and the variance, in which the common factor was extracted using the Kaiser criterion (Eigenvalue $>1$ ). Additionally, 3 common factors of the developed PHQ-8 were extracted, and the cumulative contribution rate was $55.103 \%$. The range of factor loading was $0.482-$ 0.802 , which was higher than the minimum standard of structural validity test $=0.4[46,48]$. In addition, three common factors for the SSS- 8 were extracted, and the cumulative contribution rate was $51.666 \%$. The range of factor loading was $0.353-0.881$. All coefficients related to factor loading for PHQ-8 and SSS- 8 are summarized in Tables 4 and 5 .

Confirmatory factor analysis: The model utilizes the values of chi-squared values $\left(x^{2}\right)$, root mean square residual (RMR), root mean square error of approximation (RMSEA), goodness-of-fit index (GFI), adjusted GFI (AGFI), comparative fitting index (CFI), Tucker-Lewis index (TLI), normed fit index (NFI), and other indicators to evaluate the fitting effect of the model. The small value of $\chi^{2}$ and GFI, AGFI, CFI, TLI, NFI $>0.9$ indicated that the model fitted well; the closer to the "l," the better. Moreover, RMR and RMSEA $<0.05$ indicated that the model fitted well, the closer to " 0 " fit, the better. The developed PHQ-8 and the SSS-8 were separately assessed by exploratory factor analysis, and three common factors

\begin{tabular}{lcccc}
\multicolumn{2}{l}{$\begin{array}{l}\text { Table } 3 \\
\text { and quality of life of FD patients }\end{array}$} & $\begin{array}{c}\text { Correlation } \\
\text { andysis }\end{array}$ & between & somatization \\
\hline Variable & $\begin{array}{l}\text { FD } \\
(\mathbf{n = 4 7 1 )}\end{array}$ & $\begin{array}{l}\text { PDS } \\
(\mathbf{n = 1 4 6 )}\end{array}$ & $\begin{array}{l}\text { EPS } \\
(\mathbf{n}=\mathbf{9 7})\end{array}$ & $\begin{array}{l}\text { Overlap } \\
(\mathbf{n}=\mathbf{2 2 8})\end{array}$ \\
\hline PHQ-8 & & & & \\
$r$ & 0.309 & 0.281 & 0.190 & 0.360 \\
$P$ & $<0.001$ & 0.001 & 0.063 & $<0.001$ \\
SSS-8 & & & & \\
$R$ & 0.281 & 0.236 & 0.197 & 0.317 \\
$P$ & $<0.001$ & 0.004 & 0.053 & $<0.001$ \\
\hline
\end{tabular}

FD functional dyspepsia, $P D S$ postprandial distress syndrome, EPS epigastric pain syndrome, PHQ-8 Patient Health Questionnaire-8, SSS-8 Somatic Symptom Scale-8 were extracted. Two scales confirmatory factor analysis presented a good three-factor model fit (Table 6).

\section{Correlation analysis of anxiety, depression, somatization, and the DSS for the developed PHQ-8 and SSS-8}

Somatization was evaluated by the developed PHQ-8 and the SSS-8. Correlation analysis showed that anxiety, depression, and somatization were positively correlated with DSS (Table 7). The correlation coefficient between DSS and somatization assessed by the SSS- 8 was higher than that of the developed PHQ-8.

\section{Linear regression analysis of the effects of anxiety, depression, somatization on the DSS}

Somatization, anxiety, and depression were taken as independent variables, while the DSS was taken as a dependent variable, and linear regression analysis was conducted by the backward elimination method. After adjusting for factors, such as gender, age, type of FD, level of education, and employment situation, depression and somatization were found as factors influencing the DSS (Table 8). The adjusted $\mathrm{R}^{2}$ for the PHQ- 8 and the SSS- 8 was 0.263 and 0.263 , respectively (all $P<0.001$ ). Also, the role of somatization was found to be more significant

\begin{tabular}{llll}
$\begin{array}{l}\text { Table } 4 \text { Exploratory factor } \\
\text { PHQ-8 }\end{array}$ & analysis for the developed \\
\hline Items & Factor $\mathbf{1}$ & Factor $\mathbf{2}$ & Factor 3 \\
\hline Feeling tired or having low energy & 0.802 & & \\
Fatigue & 0.769 & & \\
Insomnia or trouble sleeping & 0.525 & & \\
Dizziness & 0.482 & & \\
Back pain & & 0.797 & \\
Pain in arms, legs, or joints & & 0.719 & \\
Throat discomfort & & & 0.728 \\
Dry mouth & & & 0.706 \\
\hline PHQ-8 Patient Health Questionnaire-8 & & &
\end{tabular}

Table 5 Exploratory factor analysis for the SSS-8

\begin{tabular}{llll}
\hline Items & Factor 1 & Factor 2 & Factor 3 \\
\hline Back pain & 0.713 & & \\
Pain in arms, legs, or joints & 0.695 & & \\
Headaches & 0.495 & & \\
Chest pain or shortness of breath & 0.494 & & \\
Insomnia or trouble sleeping & & 0.806 & \\
Feeling tired or having low energy & & 0.767 & \\
Stomach or bowel problems & & & 0.881 \\
Dizziness & & & 0.353
\end{tabular}

SSS-8 Somatic Symptom Scale-8 
Table 6 Results of confirmatory factor analysis of the developed PHQ-8 and the SSS-8

\begin{tabular}{lllllllllll}
\hline Scale & Model & $\boldsymbol{x}^{\mathbf{2}}$ & df & RMR & RMSEA & GFI & AGFI & CFI & TLI & NFI \\
\hline PHQ-8 & 3 factors & 31.247 & 17 & 0.016 & 0.042 & 0.984 & 0.967 & 0.958 & 0.931 & 0.915 \\
SSS-8 & 3 factors & 17.251 & 17 & 0.010 & 0.007 & 0.991 & 0.981 & 0.998 & 0.997 & 0.927 \\
\hline
\end{tabular}

PHQ-8 Patient Health Questionnaire-8, SSS-8 Somatic Symptom Scale-8, RMR root mean square residual, RMSEA root mean square error of approximation, GFI goodness-of-fit index, AGFI adjusted goodness-of-fit index, CFI comparative fit index, TLITucker-Lewis index, NFI normed fit index

Table 7 Correlation analysis of somatization, anxiety, depression, and the dyspepsia symptom severity

\begin{tabular}{lrrrr}
\hline Variable & $\begin{array}{l}\text { FD } \\
(\mathbf{n}=612)\end{array}$ & $\begin{array}{l}\text { PDS } \\
(\mathbf{n}=\mathbf{1 7 5})\end{array}$ & $\begin{array}{l}\text { EPS } \\
(\mathbf{n}=\mathbf{1 1 2})\end{array}$ & Overlap $(\mathbf{n = 3 2 5})$ \\
\hline Anxiety & & & & \\
R & 0.223 & 0.231 & 0.147 & 0.232 \\
P & $<0.001$ & $<0.001$ & $<0.001$ & $<0.001$ \\
Depression & & & & \\
R & 0.323 & 0.296 & 0.322 & 0.333 \\
P & $<0.001$ & $<0.001$ & $<0.001$ & $<0.001$ \\
Somatization & & & & \\
$\quad$ PHQ-8 & & & & \\
R & 0.374 & 0.396 & 0.293 & 0.346 \\
P & $<0.001$ & $<0.001$ & $<0.001$ & $<0.001$ \\
Somatization & & & & \\
$\quad$ SSS-8 & & & & \\
R & 0.399 & 0.395 & 0.281 & 0.362 \\
P & $<0.001$ & $<0.001$ & $<0.001$ & $<0.001$ \\
\hline
\end{tabular}

FD functional dyspepsia, $P D S$ postprandial distress syndrome, EPS epigastric pain syndrome, $P H Q-8$ Patient Health Questionnaire-8, SSS-8 Somatic Symptom Scale-8

Table 8 Effects of somatization, anxiety, and depression on the dyspepsia symptom severity

\begin{tabular}{|c|c|c|c|c|}
\hline Variable & $\begin{array}{l}\text { Unstandardized } \\
\beta\end{array}$ & $\begin{array}{l}\text { Standardized } \\
\beta\end{array}$ & t-value & $P$ value \\
\hline Depression & 0.144 & 0.176 & 3.911 & $<0.001$ \\
\hline $\begin{array}{l}\text { Somatization } \\
\text { PHQ-8 }\end{array}$ & 0.287 & 0.256 & 6.460 & $<0.001$ \\
\hline Depression & 0.154 & 0.188 & 4.218 & $<0.001$ \\
\hline $\begin{array}{l}\text { Somatization } \\
\text { SSS-8 }\end{array}$ & 0.206 & 0.257 & 6.431 & $<0.001$ \\
\hline
\end{tabular}

PHQ-8 Patient Health Questionnaire-8, SSS-8 Somatic Symptom Scale-8

than that of depression. The standardized $\beta$ for the SSS- 8 seemed to be higher than that for the PHQ-8.

\section{Correlation analysis of anxiety, depression, somatization,} and QoL

Correlation analysis showed that somatization, anxiety and depression were positively correlated with the NDI (Table 9). Compared to the SSS-8, the correlation
Table 9 Correlation analysis of somatization, anxiety, depression and the Nepean Dyspepsia Index

\begin{tabular}{lcccc}
\hline Variable & $\begin{array}{l}\text { FD } \\
(\mathbf{n = 6 1 2})\end{array}$ & $\begin{array}{l}\text { PDS } \\
(\mathbf{n}=\mathbf{1 7 5})\end{array}$ & $\begin{array}{l}\text { EPS } \\
\mathbf{( n = 1 1 2 )}\end{array}$ & $\begin{array}{l}\text { Overlap } \\
\mathbf{( n = 3 2 5 )}\end{array}$ \\
\hline Anxiety & & & & \\
R & 0.327 & 0.258 & 0.268 & 0.367 \\
P & $<0.001$ & $<0.001$ & $<0.001$ & $<0.001$ \\
Depression & & & & \\
R & 0.354 & 0.266 & 0.304 & 0.394 \\
P & $<0.001$ & $<0.001$ & $<0.001$ & $<0.001$ \\
Somatization PHQ-8 & & & & \\
R & 0.404 & 0.325 & 0.309 & 0.446 \\
P & $<0.001$ & $<0.001$ & $<0.001$ & $<0.001$ \\
Somatization SSS-8 & & & & \\
R & 0.389 & 0.285 & 0.345 & 0.418 \\
P & $<0.001$ & $<0.001$ & $<0.001$ & $<0.001$ \\
\hline PHQ-8 Patient Health Questionnaire-8, SSS-8 Somatic Symptom Scale-8
\end{tabular}

coefficient between NDI and somatization for the developed PHQ-8 was higher.

\section{Linear regression analysis of the effects of anxiety, depression, and somatization on QoL}

The NDI for QoL was taken as a dependent variable, and somatization, anxiety, and depression were independent variables. The linear regression analysis was undertaken by backward elimination method. After adjusting for factors, such as gender, age, type of FD, level of education, and employment, situation, somatization, anxiety, and depression were noted as factors influencing the QoL (Table 10 ). The adjusted $\mathrm{R}^{2}$ for the PHQ- 8 and the SSS-8 was 0.224 and 0.236 , respectively (all $P<0.001$ ). Somatization appeared to be more important than anxiety and depression. The standardized $\beta$ for the PHQ- 8 was greater than that for the SSS-8.

\section{Discussion}

The FD is the result of the interaction of physiological function and psychosocial factors through brain-gut axis changes $[2,5]$. Somatization in psychosocial factors influences the health-related QoL after controlling the symptoms of anxiety and depression disorders in FD patients $[30,49]$. These factors may lead to the deterioration of 


\begin{tabular}{|c|c|c|c|c|}
\hline Variable & $\begin{array}{l}\text { Unstandardized } \\
\beta\end{array}$ & $\begin{array}{l}\text { Standardized } \\
\beta\end{array}$ & t-value & $P$ value \\
\hline Anxiety & 0.567 & 0.163 & 3.784 & $<0.001$ \\
\hline Depression & 0.490 & 0.136 & 3.986 & 0.003 \\
\hline $\begin{array}{l}\text { Somatization } \\
\text { (PHQ-8) }\end{array}$ & 1.330 & 0.270 & 6.638 & $<0.001$ \\
\hline Anxiety & 0.541 & 0.156 & 3.580 & $<0.001$ \\
\hline Depression & 0.557 & 0.155 & 3.416 & 0.001 \\
\hline $\begin{array}{l}\text { Somatization } \\
\text { (SSS-8) }\end{array}$ & 0.883 & 0.250 & 6.311 & $<0.001$ \\
\hline
\end{tabular}

PHQ-8 Patient Health Questionnaire-8, SSS-8 Somatic Symptom Scale-8

symptoms by aggravating anxiety and depression, resulting in repeated medical treatment and economic burden [33]. Somatization symptoms are extensively assessed by PHQ-15, possessing high reliability and validity not only in general population, but also in patient population [34, 50].

However, the assessment of somatization symptoms by the PHQ-15 exhibits several limitations. First, the PHQ15 is not a specific scale for FD patients, which has three items overlapping with gastrointestinal symptoms. Secondly, there is a menstrual problem which is not applicable to menopausal women and may cause differences in gender analysis. Thirdly, due to cultural differences, patients assessed by the PHQ- 15 have a poor compliance with questions related to sexual issues. Fourthly, the relative incidence of syncopal items is low [35]. Finally, the majority of patients with somatic symptoms are often examined in a general outpatient clinic without non-psychiatric doctors, which might result in less satisfactory results. Hence, it is highly essential to use a simple tool to assess the FD patients' somatization symptoms and severity, which is conducive to doctors as they can advise the patients about reliable therapeutic choices.

Therefore, the SSS- 8 has been used for fast assessment of somatization symptoms and its severity, avoiding differences in gender caused by menstrual abnormalities, sexual life problems caused by cultural differences, and low incidence of syncopal items [38], while it is a nonspecific scale for FD patients and overlaps with gastrointestinal symptoms in the clinical application. In addition, the SSS-8 did not include FD patients' typical symptoms, such as throat pain, which might lead to neglect those symptoms by clinicians.

Hence, due to limitations of the SSS-8, in the present study, the PHQ- 8 was developed by screening 25 items related to somatization. Gierk et al. [40] used confirmatory factor analysis to analyze the validity of the SSS-8, and the results showed that the 3-factor model outperformed the 1-factor model. Similarly, in the current study, we used a 3-factor model for confirmatory analysis of the PHQ-8 and the SSS-8. The 3-factor model fitted for the SSS-8 showed a superior performance than that of the PHQ-8.

However, the developed PHQ-8 was superior to the SSS- 8 for FD patients as follows: firstly, the PHQ- 8 is a specific scale for FD patients; the PHQ-8 includes typical symptoms of somatization, such as throat pain, which has no overlap with gastrointestinal symptoms. Secondly, in the PHQ-8, each item is significantly correlated with the total score, reflecting a higher reliability of the PHQ-8 than that of the SSS-8. Thirdly, the PHQ-8 may be superior than the SSS- 8 in the internal consistency. Fourthly, the PHQ-8 has a higher cumulative contribution rate than the SSS-8 in the exploratory factor analysis. Finally, the PHQ-8 is markedly correlated with NDI as compared to SSS-8, when NDI is used as a classic QoL assessment scale in the criterion validity.

The developed PHQ-8 was further evaluated in order to compare with the SSS-8 for 612 FD patients, including anxiety or depression, because previous studies showed that anxiety, depression, and somatization had mutual effects [21,33]. The results of the present study unveiled that the SSS-8 might play a significant role in the DSS than the PHQ- 8 because of the fact that the SSS- 8 overlaps with gastrointestinal symptoms. However, somatization assessed by the PHQ- 8 may have a greater influence on QoL than that of the SSS-8 in FD patients.

Nevertheless, the present has some limitations. Firstly, the patients with FD came from tertiary hospitals. These patients may have severe symptoms, limiting the prevalence of other people with FD. However, whether other FD patients from the community can achieve the same conclusion requires further investigation. Secondly, this study mainly analyzed and evaluated specific somatization symptoms of FD patients by the developed PHQ8; the cohort did not involve healthy individuals and patients with other diseases (irritable bowel syndrome). Thirdly, the PHQ-8 was not tested for retesting reliability in all the cases; thus, the reliability of the scales needs to be further confirmed. Fourthly, similar to the SSS-8, the PHQ- 8 was used to assess the severity of somatoform disorders [34]. Finally, the data obtained via questionnaires were often abnormally distributed, while those similar to normally distributed data were reported in previous large-sample studies [33, 44]. Accordingly, further studies with large sample size are required to improve the rationality of the developed PHQ-8.

In presence of the above-mentioned limitations, the results of the present study showed that the PHQ-8 developed for evaluating somatization and QoL 
outperformed the SSS-8 in FD patients not only without but also with anxiety and depression. All patients self-completed the PHQ-8. Somatization may be a major factor influencing DSS and NDI as compared to anxiety and depression, as well as $H$. pylori infection, gastric sensitivity, gastric accommodation, and gastric emptying in FD patients [21, 34]. Somatization is mainly treated by antidepressant drugs, which were often used to treat functional dyspepsia in patients who do not exhibit the effects of combined prokinetic and proton pump inhibitor therapy $[34,51]$. Therefore, evaluating somatization is essential in the study of FD patients; also, PHQ-8 might be applied to assess the effects of antidepressant drugs to treat functional dyspepsia. Therefore, PHQ-8 might be valuable in assessing somatization using SSS- 8 in the future FD-based studies.

\section{Conclusions}

The PHQ-15 and the SSS-8 have overlap in gastrointestinal symptoms in FD patients, and the SSS-8 was recommended to be a replacement for the PHQ-15 in a short period of time. The developed PHQ-8 without having overlap in gastrointestinal symptoms showed to have satisfactory reliability and validity. Somatization assessed by the developed PHQ-8 may have a greater impact on QoL of FD patients compared with the SSS-8. Our findings suggested that the developed PHQ- 8 may be more effective for evaluating the influences of somatization on QoL of FD patients rather than SSS-8, which may be helpful to further verify the efficacy of the developed PHQ-8 to improve a more reliable assessment for the effects of somatization on FD patients.

\begin{abstract}
Abbreviations
FD: Functional dyspepsia; SSS-8: Somatic Symptom Scale-8; PHQ-8: Patient Health Questionnaire-8; QoL: Quality of life; NDI: Nepean Dyspepsia Index; RMR: Root mean square residual; RMSEA: Root mean square error of approximation; GFI: Goodness-of-fit index; AGFI: Adjusted goodness-of-fit index; CFI: Comparative fit index; TLI: Tucker-Lewis index; NFI: Normed fit index; EPS: Epigastric pain syndrome; PDS: Postprandial distress syndrome; PHQ-15: Patient Health Questionnaire-15; PHQ-12: Patient Health Questionnaire-12; GAD-7: Generalized Anxiety Disorder-7; PHQ-9: Patient Health Questionnaire-9; DSS: Dyspepsia symptom severity.
\end{abstract}

\section{Acknowledgements}

None.

\section{Authors' contributions}

YCQ and YGZ designed the study, analyzed and interpreted the data, and drafted the manuscript; WX and XT collected and analyzed the data, and drafted the manuscript; WCY and YY participated in data analysis and edited the manuscript; HGB participated in the study design, analyzed the data, and revised the manuscript. All authors read and approved the final manuscript.

\section{Funding}

None.

\section{Availability of data and materials}

The data that support the findings of this study are available from the corresponding author upon a reasonable request.

\section{Ethics approval and consent to participate}

This study was approved by the Ethics Committee of the Affiliated Hospital of North Sichuan Medical College (Nanchong, China). All the patients signed the written informed consent form.

\section{Consent for publication \\ Not applicable.}

\section{Competing interests}

The authors declare that they have no competing interests.

\section{Author details}

${ }^{1}$ Department of Gastroenterology, Affiliated Hospital of North Sichuan Medical College, No. 67 Wenhua Road, Shunqing, Nanchong 637000, Sichuan, China. ${ }^{2}$ Department of Nursing, North Sichuan Medical College, No. 234 Fujiang Road, Shunqing, Nanchong 637000, Sichuan, China.

Received: 23 April 2020 Accepted: 19 October 2020

Published online: 28 October 2020

\section{References}

1. Talley NJ, Stanghellini V, Chan FKL, and The Rome IV Committees. Gastroduodenal disorders. Rome IV functional gastrointestinal disorders-disorders of gut-brain interaction, Drossman DA, Chang L, Kellow J, Tack J, Whitehead WE, ed. Raleigh, NC: The Rome Foundation, 2016, 903-935.

2. Drossman DA. Functional gastrointestinal disorders: history, pathophysiology, clinical features, and Rome IV. Gastroenterology. 2016;150(6):1262-79.

3. Kabakambira JD, Hategeka C, Page C, Ntirenganya C, Dusabejambo V, Ndoli J, Ngabonziza F, Hale D, Bayingana C, Walker T. Efficacy of Helicobacter pylori eradication regimens in Rwanda: a randomized controlled trial. BMC Gastroenterol. 2018;18(1):134-43.

4. Talley NJ, Walker MM, Holtmann G. Functional dyspepsia. Currt Opin Gastroenterol. 2016;32(6):467-73.

5. Du LJ, Chen BR, Kim JJ, Kim S, Shen JH, Dai N. Helicobacter pylori eradication therapy for functional dyspepsia: systematic review and meta-analysis. World J Gastroenterol. 2016;22(12):3486-95.

6. Mearin F, Pérez-Oliveras M, Perelló A, Vinyet J, Ibañez A, Coderch J, et al. Dyspepsia and irritable bowel syndrome after a Salmonella gastroenteritis outbreak: one-year follow-up cohort study. Gastroenterology. 2005;129(1):98-104.

7. Göktaş Z, Köklü S, Dikmen D, Öztürk Ö, Yılmaz B, Asıl M, et al. Nutritional habits in functional dyspepsia and its subgroups: a comparative study. Scand J Gastroenterol. 2016;51(8):903-7.

8. Jiang SM, Lei XG, Jia L, Xu M, Wang SB, Liu J, et al. Unhealthy dietary behavior in refractory functional dyspepsia: a multicenter prospective investigation in China. J Digest Dis. 2014;15(12):654-9.

9. Miwa H, Nakajima K, Yamaguchi K, Fujimoto K, Veldhuyzen VAN, Zanten SJ. Generation of dyspeptic symptoms by direct acid infusion into the stomach of healthy Japanese subjects. Aliment Pharmacol Ther. 2007;26(2):257-64.

10. Shi H, Zhu S, Qin B, Wang L, Yang J, Lu G, Dai F. Nerve growth factor and Tropomyosin receptor kinase A are increased in the gastric mucosa of patients with functional dyspepsia. BMC Gastroenterol. 2019;19(1):221-9.

11. Vijayvargiya P, Camilleri M, Chedid V, Mandawat A, Erwin PJ, Murad $\mathrm{MH}$. Effects of promotility agents on gastric emptying and symptoms: a systematic review and meta-analysis. Gastroenterology. 2019;156(6):1650-60.

12. Karamanolis G, Caenepeel P, Arts J, Tack J. Association of the predominant symptom with clinical characteristics and pathophysiological mechanisms in functional dyspepsia. Gastroenterology. 2006;130(2):296-303.

13. Vanheel $H$, Carbone F, Valvekens L, Simren M, Tornblom H, Vanuytsel T, et al. Pathophysiological abnormalities in functional dyspepsia subgroups according to the Rome III criteria. Am J Gastroenterol. 2017;112(1):132-40. 
14. Farré $R$, Vanheel $H$, Vanuytsel T, Masaoka T, Törnblom H, Simrén $M$, et al. In functional dyspepsia, hypersensitivity to postprandial distention correlates with meal-related symptom severity. Gastroenterology. 2013;145(3):566-73.

15. Simren M, Tornblom H, Palsson OS, van Tilburg MAL, Van Oudenhove $L$, Tack J, et al. Visceral hypersensitivity is associated with Gl symptom severity in functional $\mathrm{Gl}$ disorders: consistent findings from five different patient cohorts. Gut. 2018;67(2):255-62.

16. Hammer J. Identification of individuals with functional dyspepsia with a simple, minimally invasive test: a single center cohort study of the oral capsaicin test. Am J Gastroenterol. 2018;113(4):584-92.

17. Futagami S, Itoh T, Sakamoto C. Systematic review with meta-analysis: post-infectious functional dyspepsia. Aliment Pharmacol Ther. 2015;41(2):177-88.

18. Jung $\mathrm{H}$, Talley NJ. Role of the duodenum in the pathogenesis of functional dyspepsia: a paradigm shift. J Neurogastroenterol Motil. 2018;24(3):345-54.

19. Hwang SW, Kim N, Jung H, Park JH, Choi YJ, Kim H, et al. The association of SLC6A4 5-HTTLPR and TRPV1 $945 \mathrm{G}>\mathrm{C}$ with functional dyspepsia in Korea. J Gastroenterol Hepatol. 2014;29(10):1770-7.

20. Singh R, Mittal B, Ghoshal UC. Functional dyspepsia is associated with GNbeta3 C825T and CCK-ART/C polymorphism. Eur J Gastroenterol Hepatol. 2016;28(2):226-32.

21. Van Oudenhove L, Vandenberghe J, Geeraerts B, Bolino C, Pintos-Sanchez MI, Moayyedi $P$, et al. Determinants of symptoms in functional dyspepsia: gastric sensorimotor function, psychosocial factors or somatisation? Gut. 2008;57(12):1666-73.

22. Gracie DJ, Bercik P, Morgan DG, Bolino C, Pintos-Sanchez MI, Moayyedi $P$, et al. No increase in prevalence of somatization in functional vs organic dyspepsia: a cross-sectional survey. Neurogastroenterol Motil. 2015;27(7):1024-31

23. Clauwaert N, Jones MP, Holvoet L, Vandenberghe J, Vos R, Tack J, et al. Associations between gastric sensorimotor function, depression, somatization, and symptom-based subgroups in functional gastroduodenal disorders: are all symptoms equal? Neurogastroenterol Motil. 2012;24(12):1088-e565.

24. Lee SP, Sung I, Kim JH, Lee SY, Park HS, Shim CS. The effect of emotional stress and depression on the prevalence of digestive diseases. J Neurogastroenterol Motil. 2015;21 (2):273-82.

25. Bulut CB, Ozkula G, Isikli S, Özkan Göncüoğlu İ, Öcal S, Alınöz AE, et al. Anxiety, depression, and anger in functional gastrointestinal disorders: a cross-sectional observational study. Psychiatry Res. 2018;268(3):368-72.

26. Hanel G, Henningsen P, Herzog W, Sauer N, Schaefert R, Szecsenyi J, et al. Depression, anxiety, and somatoform disorders: vague or distinct categories in primary care? Results from a large cross-sectional study. J Psychosom Res. 2009;67(3):189-97.

27. MacLean EW, Palsson OS, Turner MJ, Whitehead WE. Development and validation of new disease-specific measures of somatization and comorbidity in IBS. J Psychosom Res. 2012;73(5):351-5.

28. Barsky AJ. Assessing the New DSM-5 diagnosis of somatic symptom disorder. Psychosom Med. 2016;78(1):2-4.

29. Toussaint A, Kroenke K, Baye F, Lourens S. Comparing the Patient Health Questionnaire-15 and the Somatic Symptom Scale-8 as measures of somatic symptom burden. J Psychosom Res. 2017;101(1):44-50.

30. Lipowski ZJ. Somatization: the concept and its clinical application. Am J Psychiatry. 1988;145(11):1358-68.

31. Vu J, Kushnir V, Cassell B, Gyawali CP, Sayuk GSL. The impact of psychiatric and extraintestinal comorbidity on quality of life and bowel symptom burden in functional GI disorders. Neurogastroenterol Motil. 2014;26(9):1323-32.

32. Pintosanchez MI, Ford AC, Avila CA, Verdu EF, Collins SM, Morgan D, et al. Anxiety and depression increase in a stepwise manner in parallel with multiple FGIDs and symptom severity and frequency. Am J Gastroenterol. 2015;110(7):1038-48
33. Kindt S, Van Oudenhove L, Mispelon L, Caenepeel P, Arts J, Tack J. Longitudinal and cross-sectional factors associated with long-term clinical course in functional dyspepsia: a 5-year follow-up study. Am J Gastroenterol. 2011;106(2):340-8.

34. Kroenke K, Spitzer RL, Williams JB. The PHQ-15: validity of a new measure for evaluating the severity of somatic symptoms. Psychosom Med. 2002;64(2):258-66

35. Spiller RC, Humes DJ, Campbell E, Hastings M, Neal KR, Dukes GE, et al. The Patient Health Questionnaire 12 Somatic Symptom scale as a predictor of symptom severity and consulting behaviour in patients with irritable bowel syndrome and symptomatic diverticular disease. Aliment Pharmacol Ther. 2010;32(6):811-20.

36. Zhang L, Fritzsche K, Liu Y, Wang J, Huang M, Wang Y, et al. Validation of the Chinese version of the PHQ-15 in a tertiary hospital. BMC Psychiatry. 2016;16(1):1-9.

37. Narrow WE, Clarke DE, Kuramoto SJ, Kraemer HC, Kupfer DJ, Greiner L, et al. DSM-5 field trials in the United States and Canada, Part III: development and reliability testing of a cross-cutting symptom assessment for DSM-5. Am J Psychiatry. 2013;17(1):71-82.

38. Gierk B, Kohlmann S, Kroenke K, Spangenberg L, Zenger M, Brähler E, et al. The Somatic Symptom Scale-8 (SSS-8):a brief measure of somatic symptom burden. JAMA Intern Med. 2014;174(3):399-407.

39. Matsudaira K, Oka H, Kawaguchi M, Murakami M, Fukudo S, Hashizume $\mathrm{M}$, et al. Development of a Japanese version of the Somatic Symptom Scale-8: psychometric validity and internal consistency. Gen Hosp Psychiatry. 2017:45(1):7-11.

40. Gierk B, Kohlmann S, Toussaint A, Wahl I, Brünahl CA, Murray AM, et al. Assessing somatic symptom burden: a psychometric comparison of the Patient Health Questionnaire-15 (PHQ-15) and the Somatic Symptom Scale-8 (SSS-8). J Psychol Res. 2015;78(4):352-5.

41. Creswell JW. Educational research: planning, conducting, and evaluating quantitative and qualitative research. 4th ed. Upper Saddle River, NJ: Pearson Education Inc:; 2012, 147, 609-611.

42. Spitzer RL, Kroenke K, Williams JB, Löwe BL. A brief measure for assessing generalized anxiety disorder: the GAD-7. Arch Intern Med. 2006;166(10):1092-7.

43. Kroenke K, Spitzer RL, Williams JB. The PHQ-9: validity of a brief depression severity measure. J Gen Intern Med. 2001;16(9):606-13.

44. Talley NJ, Verlinden M, Jones M. Quality of life in functional dyspepsia: responsiveness of the Nepean Dyspepsia Index and development of a new 10-item short form. Aliment Pharmacol Ther. 2001;15(2):207-16.

45. Plummer F, Manea L, Trepel D, McMillan D. Screening for anxiety disorders with the GAD-7 and GAD-2: a systematic review and diagnostic meta analysis. Gen Hosp Psychiatry. 2016;39(1):24-31.

46. Floyd FJ, Widaman KF. Factor analysis in the development and refinement of clinical assessment instruments. Psychol Assess. 1995;7(3):286-99.

47. Cronbach LJ. Coefficient alpha and the internal structure of tests. Psychometrika. 1951;16(2):297-334.

48. Raykov T. Behavioral scale reliability and measurement invariance evaluation using latent variable modeling. Behav Ther. 2004;35(2):299-331.

49. Jones MP, Coppens E, Vos R, Holvoet L, Luyten P, Tack J, et al. A multidimensional model of psychobiological interactions in functional dyspepsia: a structural equation modelling approach. Gut. 2013;62(11):1573-80.

50. Zijlema WL, Stolk RP, Löwe B, Rief W, White PD, et al. How to assess common somatic symptoms in large scale studies: a systematic review of questionnaires. J Psychosom Res. 2013;74(6):459-68.

51. Ford AC, Luthra P, Tack J, Boeckxstaens GE, Moayyedi P, Talley NJ. Efficacy of psychotropic drugs in functional dyspepsia: Systematic review and meta-analysis. Gut. 2017;66(3):411-20.

\section{Publisher's Note}

Springer Nature remains neutral with regard to jurisdictional claims in published maps and institutional affiliations. 